\title{
Edge plasmons in graphene nanostructures
}

\author{
Weihua Wang, Peter Apell, and Jari Kinaret \\ Department of Applied Physics, Chalmers University of Technology, SE-412 96 Gothenburg, Sweden
}

(Received 20 April 2011; revised manuscript received 29 July 2011; published 24 August 2011)

\begin{abstract}
Plasmon modes in graphene are influenced by the unusual dispersion relation of the material. For bulk plasmons this results in a $n^{1 / 4}$ dependence of the plasma frequency on the charge density, as opposed to the $n^{1 / 2}$ dependence in two-dimensional electron gas (2DEG); yet, bulk plasmon dispersion in graphene follows a similar $q^{1 / 2}$ behavior as for other two-dimensional materials. In this work we consider finite graphene nanostructures, semi-infinite sheets, and circular disks and study edge plasmons that are confined to the boundaries of the structures. We find that, for abrupt edges, graphene edge plasmons behave analogously to those in 2DEGs, but, for gradual edge profiles, important distinctions arise. In particular, we show that for a linear edge profile, graphene supports fewer edge modes than a 2DEG at a given $q$, and the edge monopole plasmon dispersion in graphene follows a $q^{1 / 4}$ law in contrast to the $q^{0}$ behavior seen in 2DEGs.
\end{abstract}

DOI: 10.1103/PhysRevB.84.085423

PACS number(s): 73.20.Mf, 73.21.-b

\section{INTRODUCTION}

In addition to the regular bulk plasmons, ${ }^{1,2}$ a finite twodimensional electron gas (2DEG) such as a semi-infinite half-plane ${ }^{3-6}$ or a finite disk, ${ }^{7-10}$ supports edge plasmons or perimeter waves that are localized at the boundary of the two-dimensional (2D) system. ${ }^{11}$ Xia and Quinn showed that systems with gradual edge density profiles support several types of edge waves (multipole modes) ${ }^{12}$ that can be thought of as standing-wave plasmons fitting into the diffuse edge layer. This notation is very similar as 3D multipole surface plasmons ${ }^{13-18}$ that have been observed experimentally on $\mathrm{K}$, $\mathrm{Na}$, and Ag surfaces ${ }^{19,20}$ and, very recently, in graphene-coated Ir and SiC. ${ }^{21}$ Basic requirements for the existence of such higher-multipole modes include gradual edge density profile and dispersion of bulk plasmon. In a $2 \mathrm{D}$ system the bulk plasma frequency increases as the square root of the wave vector $q$, and the edge electron density profile varies over a length scale that is determined by device structure and fabrication. Hence, we expect that higher multipole edge plamons in a 2D system exist in many experimentally relevant systems.

Graphene is a monolayer of carbon atoms arranged in a hexagonal lattice and, hence, the most ideal two-dimensional material. In the past few years, it has attracted a great deal of interest both theoretically and experimentally. ${ }^{22-25}$ The main difference between graphene and a conventional 2DEG material is the electronic energy dispersion: In graphene, the valence and conductance bands touch at discrete points ( $\mathbf{K}$ and $\mathbf{K}^{\prime}$ ) at the edge of the first Brillouin zone. For undoped graphene, the Fermi energy coincides with the top of the valence band and the bottom of the conductance band so the density of states at the Fermi energy is very small and the band structure near the Fermi energy is well approximated by $\varepsilon(\mathbf{k})=\hbar v_{F}|\mathbf{k}-\mathbf{K}|$ (with a similar expression for $\mathbf{k} \approx$ $\left.\mathbf{K}^{\prime}\right)$. This unusual band structure leads to the conductivity of graphene being proportional to the square root of the charge density $\left(n^{1 / 2}\right)$ in the semiclassical limit, ${ }^{26-30}$ while it is linear $(n)$ in a 2DEG. Because of these unique properties, plasmons in graphene also have new features, for example, the density dependence of the plasma frequency $\propto n^{1 / 4}$ (Refs. 31-34) differs from the usual 2DEG $\propto n^{1 / 2}$. Furthermore, graphene has been predicted to support transverse
(TE) surface plasmons, ${ }^{30,35,36}$ intervalley plasmons, ${ }^{37}$ and a thermoplasma polariton ${ }^{38}$ and is a good candidate for optical infrared or $\mathrm{THz}$ frequencies plasmonics applications ${ }^{29,39-41}$ and quantum plasmonics devices. ${ }^{42-44}$

In this article, we investigate graphene edge plasmons in a semi-infinite sheet and a finite disk. We consider a low electron density edge layer arising from electrostatic confinement ${ }^{45}$ and approximate it by a linear profile; a similar approach has been used as in Ref. 12. Based on the semiclassical conductivity, ${ }^{29,30}$ we derive a self-consistent integral equation for the electrostatic potential of the system and solve it numerically by expanding the potential in a complete orthonormal set of functions. 5,7,12 The resulting eigenfrequencies for the wave vector $q$ represent edge plasmon modes. We show that, for a given $q$, graphene supports fewer plasmon branches than a 2DEG and the eigenfrequencies of the monopole edge plasmon in graphene have a $q^{1 / 4}$ dependence. For disk geometry, we also briefly discuss the differences between the axisymmetric $(l=0)$ mode and modes with nonzero angular momenta.

\section{GENERAL FORMULATION}

A semiclassical model for the ac electrical conductivity ${ }^{46}$ yields the Drude-like expressions

$$
\sigma(\boldsymbol{r}, \omega)=\frac{e^{2}}{m} \frac{i}{\omega+i \tau^{-1}} n(\boldsymbol{r})
$$

in a $2 \mathrm{DEG}$ and

$$
\sigma(\boldsymbol{r}, \omega)=\frac{e^{2} v_{F}}{\pi^{1 / 2} \hbar} \frac{i}{\omega+i \tau^{-1}} \sqrt{n(\boldsymbol{r})}
$$

in graphene. ${ }^{29,30}$ Here $n(\boldsymbol{r})$ is the two-dimensional groundstate electron density, $v_{F}$ is the Fermi velocity $\left(v_{F} \approx 10^{6} \mathrm{~m} / \mathrm{s}\right.$ in graphene), and $\tau$ is the relaxation time. These expressions are valid for $\omega \tau \gg 1$, which is assumed in the following calculations.

We incorporate the effects of a varying ground-state charge density near the edge using a local theory description. The edge plasmons excitations are then contained in three basic equations: $^{12}$ the Poisson equation

$$
\nabla \cdot[\epsilon \boldsymbol{E}(\boldsymbol{r})]=-e \delta n(\boldsymbol{r}),
$$


the charge conservation law

$$
\nabla \cdot \boldsymbol{j}(\boldsymbol{r})=-i e \omega \delta n(\boldsymbol{r}),
$$

and the current density expression

$$
\boldsymbol{j}(\boldsymbol{r})=\boldsymbol{\sigma}(\boldsymbol{r}, \omega) \cdot \boldsymbol{E}(\boldsymbol{r}),
$$

where $\delta n(\boldsymbol{r})$ is the electron density fluctuation (deviation from ground-state charge density) that is assumed to be harmonic $e^{-i \omega t}$ and $\epsilon$ is the background dielectric constant. In the electrostatic limit the electric field can be expressed as the gradient of a scalar potential $\Phi(\boldsymbol{r})$, and by combining Eqs. (3)-(5) a self-consistent integral equation for the potential $\Phi(\boldsymbol{r})$ is obtained.

\section{A. A semi-infinite sheet}

In a semi-infinite sheet, the electron density is uniform parallel to the edge and has a profile $n_{0} f(x)$ perpendicular to the edge, where $f(x)=0$ for $x>0$ and $f(x)=1$ for $x<-a$, and $a$ is the edge layer width. For this profile, combining with Eq. (3), Poisson's equation now becomes

$$
\nabla^{2} \Psi=\frac{e \delta n(x)}{\epsilon} \delta(z) \Theta(-x) .
$$

This equation has plane-wave solutions along the edge, proportional to $e^{i q y-i \omega t}$, whose amplitude $\Phi(x, z)$ depends on the wave vector $q$. A Fourier transformation in the $x$ direction yields the differential equation

$$
\left[\frac{\partial^{2}}{\partial z^{2}}-\left(k^{2}+q^{2}\right)\right] \bar{\Phi}(k, z)=\frac{e \delta n_{k}}{\epsilon} \delta(z),
$$

where

$$
\delta n_{k}=\frac{1}{2 \pi} \int_{-\infty}^{0} e^{-i k x} \delta n(x) d x .
$$

For $z \neq 0$, Eq. (7) reduces to a Laplace equation, and the only allowed solution is proportional to $e^{-\left(k^{2}+q^{2}\right)^{1 / 2}|z|}$. Using the boundary condition at $z=0$

$$
\left.\frac{\partial \bar{\Phi}(k, z)}{\partial z}\right|_{z=+0}-\left.\frac{\partial \bar{\Phi}(k, z)}{\partial z}\right|_{z=-0}=\frac{e}{\epsilon} \int_{-0}^{+0} \delta n_{k} \delta(z) d z,
$$

the solution in the $z=0$ plane reduces to

$$
\bar{\Phi}(k, 0)=-\frac{e \delta n_{k}}{2 \epsilon\left(k^{2}+q^{2}\right)^{1 / 2}} .
$$

An inverse Fourier transformation now gives a nonlocal integral relation between the electrostatic potential $\Phi(x, z=0)$ in the plane and the corresponding electron density fluctuation. Taking into account Eqs. (4) and (5), the electron density fluctuation is determined by the induced potential as

$$
\begin{aligned}
\delta n\left(x^{\prime}\right)= & -\frac{1}{i e \omega}\left[\sigma\left(q^{2}-\frac{d^{2}}{d x^{\prime 2}}\right) \Phi\left(x^{\prime}, z=0\right)\right. \\
& \left.-\frac{d \sigma}{d x^{\prime}} \frac{d \Phi\left(x^{\prime}, z=0\right)}{d x^{\prime}}\right]
\end{aligned}
$$

and then the self-consistent integral equation for the in-plane potential $\Phi(x, z=0)$ in a 2DEG is obtained as

$$
\begin{aligned}
\Phi(x)= & \frac{2 \omega_{a}^{2}}{\omega^{2}} \int_{-\infty}^{0} d x^{\prime} L_{q}\left(x-x^{\prime}\right) \\
& \times\left[f\left(x^{\prime}\right)\left(q^{2}-\frac{d^{2}}{d x^{\prime 2}}\right)-\frac{d f\left(x^{\prime}\right)}{d x^{\prime}} \frac{d}{d x^{\prime}}\right] \Phi\left(x^{\prime}\right),
\end{aligned}
$$

where $\Phi(x) \equiv \Phi(x, z=0)$ and $x$ is given in units of $a$ and $q$ in units of $1 / a$. As long as the width of the edge region is much larger than the effective Bohr radius $a_{0}, a \gg a_{0}$, the nonlocal effects can be neglected. ${ }^{12}$ The prefactor $\omega_{a}^{2}=e^{2} n_{0} / 2 m \epsilon a$ is the squared 2DEG bulk plasma frequency at wave vector $1 / a$. The kernel $L_{q}\left(x-x^{\prime}\right)$ is given by

$$
L_{q}\left(x-x^{\prime}\right)=\int_{-\infty}^{\infty} \frac{d k}{4 \pi} \frac{e^{i k\left(x-x^{\prime}\right)}}{\left(k^{2}+q^{2}\right)^{1 / 2}}=\frac{K_{0}\left(q\left|x-x^{\prime}\right|\right)}{2 \pi},
$$

according to the formula 9.6.21 in Ref. 47. Equation (12) can be solved numerically to determine the dispersion relations for different plasmon branches.

A similar equation can be defined for graphene by inserting the graphene conductivity in Eq. (11). This yields

$$
\begin{aligned}
\Phi(x)= & \frac{2 \omega_{a}^{2}}{\omega^{2}} \int_{-\infty}^{0} d x^{\prime} L_{q}\left(x-x^{\prime}\right)\left[\sqrt{f\left(x^{\prime}\right)}\left(q^{2}-\frac{d^{2}}{d x^{\prime 2}}\right)\right. \\
& \left.-\frac{d \sqrt{f\left(x^{\prime}\right)}}{d x^{\prime}} \frac{d}{d x^{\prime}}\right] \Phi\left(x^{\prime}\right),
\end{aligned}
$$

where $\omega_{a}^{2}=e^{2} v_{F} \sqrt{n_{0}} /(2 \sqrt{\pi} \hbar \epsilon a)$ is the squared bulk plasma frequency at wave vector $1 / a$ in graphene.

Equations (12) and (14) are standard eigenvalue problems, and for a given $q$ have nontrivial solutions only at some specific frequencies $\omega$ that correspond to edge plasmon eigenfrequencies. Interestingly, the main difference between the equations for a 2DEG and graphene is the different power of the edge profile $f(x)$ that appears in Eqs. (12) and (14). This implies that for an abrupt edge, which is described by $f(x)=0$ or $f(x)=1$, there is no difference between a 2DEG and graphene as $f(x)=\sqrt{f(x)}$ (except for the differences arising from the prefactors $\omega_{a}^{2}$ ), and the edge plasmon dispersion relation is approximately given by $\omega(q)=(2 / 3)^{1 / 2} \omega_{B}(q)$, where $\omega_{B}(q)$ is the bulk plasma frequency. ${ }^{12}$ For any nonabrupt edge profile, edge plasmons in a $2 \mathrm{DEG}$ and graphene will behave differently.

For a general edge profile, Eqs. (12) and (14) cannot be solved analytically, and an appropriate numerical calculation is needed. We expand the potential $\Phi(x)$ in Laguerre polynomials $5,12,47$

$$
\Phi(x)=\exp (q x) \sum_{n=0}^{\infty} c_{n} L_{n}(-2 q x)
$$

for $x<0$ and transform the integral equation into a matrix equation. The numerical calculation must include a sufficient number of terms to guarantee convergence; we find that the results converge quickly and $n=20$ yields the desired accuracy both for Eqs. (12) and (14). The potential at $x>0$ can be obtained from Eqs. (12) or (14) once the potential for $x<0$ is known. 


\section{B. A finite disk}

We model a finite disk of radius $R$ with an electron profile $n_{0} f(r)$, where $f(r)=0$ for $r>R$ and $f(r)=1$ for $r<R-$ $a$, and $a$ is the edge layer width. In the following calculation we use dimensionless parameters, with length in units of $R$ and momenta in units of $1 / R$. Due to cylindrical symmetry, the solutions of Eq. (3) are proportional to $e^{i l \psi-i \omega t}$ with the amplitude $\Phi(r, z)$ depending on angular momenta $l$. The same technique can be used as in the semi-infinite case, using the Hankel transformation ${ }^{7}$ instead of the Fourier transformation, and we obtain for a $2 \mathrm{DEG}$

$$
\begin{aligned}
\Phi(r)= & \frac{2 \omega_{R}^{2}}{\omega^{2}} \int_{0}^{1} d r^{\prime} K\left(r, r^{\prime}\right) r^{\prime}\left[f\left(r^{\prime}\right)\left(\frac{l^{2}}{r^{\prime 2}}-\frac{1}{r^{\prime}} \frac{\partial}{\partial r^{\prime}} r^{\prime} \frac{\partial}{\partial r^{\prime}}\right)\right. \\
& \left.-\frac{\partial f\left(r^{\prime}\right)}{\partial r^{\prime}} \frac{\partial}{\partial r^{\prime}}\right] \Phi\left(r^{\prime}\right),
\end{aligned}
$$

where the kernel is

$$
K\left(r, r^{\prime}\right)=\frac{1}{2} \int_{0}^{\infty} d p J_{l}(p r) J_{l}\left(p r^{\prime}\right) .
$$

For graphene the corresponding integral equation is

$$
\begin{aligned}
\Phi(r)= & \frac{2 \omega_{R}^{2}}{\omega^{2}} \int_{0}^{1} d r^{\prime} K\left(r, r^{\prime}\right) r^{\prime}\left[\sqrt{f\left(r^{\prime}\right)}\left(\frac{l^{2}}{r^{\prime 2}}-\frac{1}{r^{\prime}} \frac{\partial}{\partial r^{\prime}} r^{\prime} \frac{\partial}{\partial r^{\prime}}\right)\right. \\
& \left.-\frac{\partial \sqrt{f\left(r^{\prime}\right)}}{\partial r^{\prime}} \frac{\partial}{\partial r^{\prime}}\right] \Phi\left(r^{\prime}\right) .
\end{aligned}
$$

The parameter $\omega_{R}$ has the same definition as $\omega_{a}$ above.

We expand the potential in orthonormal polynomials on our integral interval, using Jacobi polynomials $P_{n}^{l, 0}\left(1-2 r^{2}\right){ }^{7,47}$ as

$$
\Phi(r)=\sum_{n=0}^{\infty} c_{n} r^{l} P_{n}^{(l, 0)}\left(1-2 r^{2}\right)
$$

for $r<1$. We then transform the above integral equation into a matrix equation and find the eigenfrequencies $\omega_{l}$ of the system. In parallel with our analysis of the semi-infinite sheet, we truncate the series at $n=10$.

\section{NUMERICAL RESULTS}

In this part we present the numerical results for linear edge profiles for semi-infinite sheets and finite disks, where the electron densities linearly decay from bulk to zero inside the edge layer. The linear edge profile is a good approximation to systems with electrostatic confinement using gate electrodes, and in this case the edge layer width is roughly given by the gate oxide thickness. For more general edge profile the details of the solution will differ but the qualitative features remain.

\section{A. A semi-infinte sheet}

We have calculated the plasmon dispersions in a 2DEG and in graphene, shown in Fig. 1. The 2DEG results have been previously obtained by Xia and Quinn. ${ }^{12}$ We see that with the same linear edge profile the edge plasmons differ completely in graphene and in a 2DEG, which is related to the difference in conductivity of Eqs. (1) and (2). The 2DEG is seen to support more modes than graphene at the same wave vector; for instance, for $q a=3$, there are four modes (monopole and

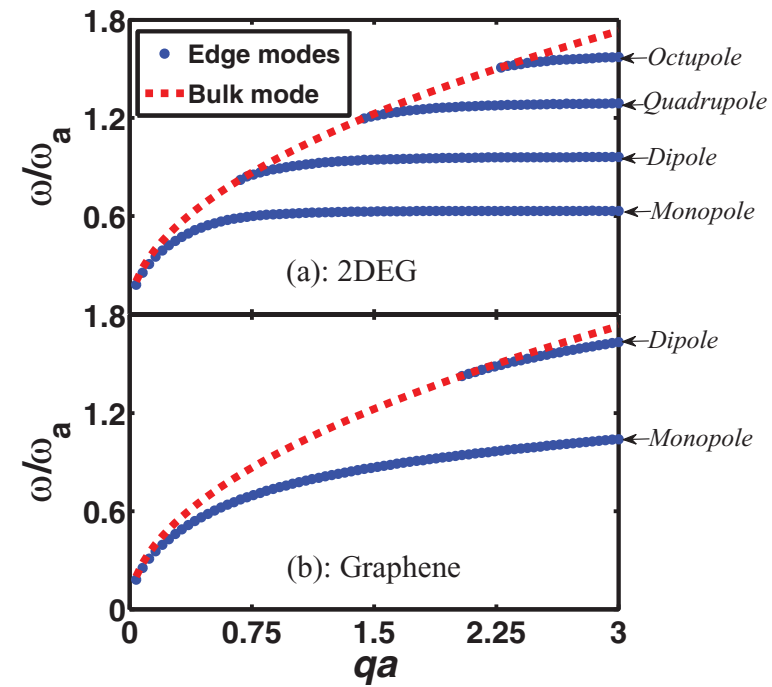

FIG. 1. (Color online) Edge plasmon dispersions for (a) 2DEG and (b) graphene calculated from Eqs. (12) and (14), respectively. In the $2 \mathrm{DEG}$, up to four edge plasmon branches are seen in this range of wave vectors, while only two branches are supported by graphene. The dispersion relations in the 2DEG flatten out quite quickly and approach constants for large $q$ while in graphene the plasma frequencies continue to increase with increasing $q$.

three higher multipole modes) in a 2DEG, while only the monopole and the dipole modes are supported in graphene.

The dispersion relations for the different edge modes also differ, being nearly flat for large $q a$ in 2DEG but having an apparent slope in graphene. Especially for the monopole mode this can be understood by inspecting the self-consistent integral equations and their solutions. The numerical solution for the leading coffecient $c_{0}$ is shown in Fig. 2 as a function of the wave vector [the expansion (15) is normalized so that $\sum\left|c_{n}\right|^{2}=1$ ]. We see that the expansion is dominated by the first coefficient, and $\Phi(x) \approx e^{q x}$ for $x<0$ [note that $L_{0}(-2 q x)=1$ ]. Inserting this back into Eqs. (12) and (14), we see that the first term $\left(q^{2}-d^{2} / d x^{\prime 2}\right) \Phi\left(x^{\prime}\right)$ equals to zero, and only the second term contributes. The second term contains the derivative of the conductivity, which is not zero only in the edge layer. So at this limit the monopole localizes only at the edge layer and only this part of electrons contributes to the plasmon resonance.

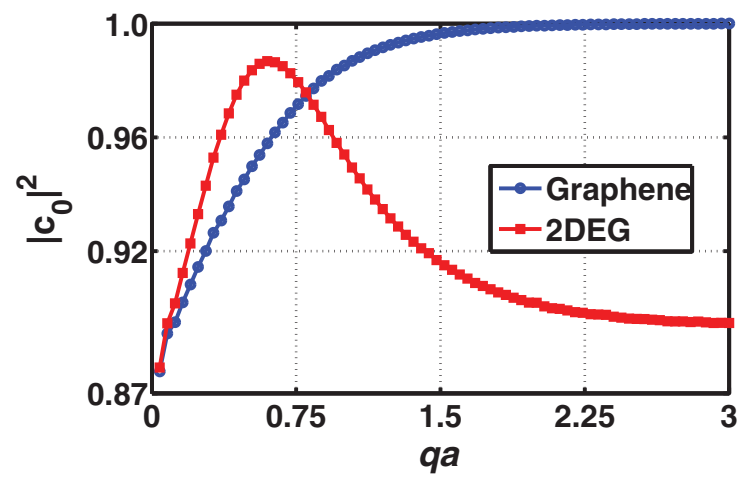

FIG. 2. (Color online) The first term $c_{0}$ coefficient in the potential of the monopole mode for 2DEG and graphene. It is almost $90 \%$ in 2DEG and exact 1 in graphene for $q a>1.5$. 
This argument is exact to graphene but only approximate for the 2DEG. The integral equation for a $2 \mathrm{DEG}$ now yields

$$
\Phi(y)=\frac{2 \omega_{a}^{2}}{\omega^{2}} \int_{-q a}^{0} d y^{\prime} L\left(y-y^{\prime}\right) \exp \left(y^{\prime}\right),
$$

where we have set $y=q x$. This can be numerically evaluated and, considering the potential being continuous at the boundary $x=0$, directly gives

$$
\lim _{q \rightarrow \infty} \omega=0.56 \omega_{a} .
$$

The limit is approached quite rapidly and the dispersion is essentially flat for $q a \gtrsim 1.5$. This is a little lower than the numerical result $0.6 \omega_{a}$, because there are contributions from other $c_{n}$ polynomial terms, which means there are also a little contributions from the electrons out of the edge layer. For graphene, the integral equation gives

$$
\Phi(y)=\frac{2 \omega_{a}^{2}}{\omega^{2}} \sqrt{q a} \int_{-q a}^{0} d y^{\prime} \frac{1}{2 \sqrt{-y^{\prime}}} L\left(y-y^{\prime}\right) \exp \left(y^{\prime}\right),
$$

which results in

$$
\omega \sim 0.79 \omega_{a}(q a)^{1 / 4}
$$

for $q a \rightarrow \infty$; the expression is quite accurate for $q a \gtrsim 1.5$, where the potential is exactly only the first term $c_{0}$ (cf. Fig. 2).

Hence, in the regime $q a \gtrsim 1.5$, the monopole plasma frequency is proportional to $q^{1 / 4}$ in graphene and to $q^{0}$ in 2DEG. In Fig. 3 we plot $\omega /(q a)^{1 / 4}$ for the monopole mode based on the numerical solution of Eq. (14). We see that the numerical results are in good agreement with the analytic argument.

\section{B. A finite disk}

For a finite disk geometry we use the numerical method introduced by Fetter ${ }^{7}$ for a disk with an abrupt edge profile; as we know, 2DEG edge plasmons have not been previously analyzed for this structure with linear profiles. There are some references ${ }^{45,48}$ considering the plasmon resonances of a disk geometry with other specific edge profiles. An approximate oblate spheroid coordinate system was used to describe a disk geometry by letting the out-of-plane direction approach zero.

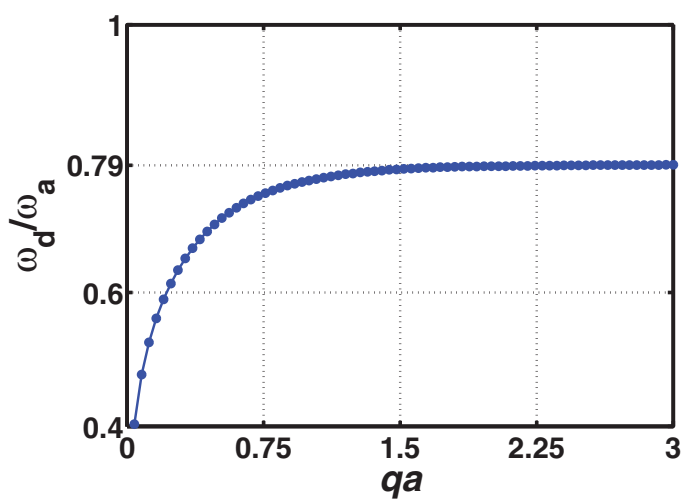

FIG. 3. (Color online) The frequencies $\omega_{d}=\omega /(q a)^{1 / 4}$ numerically calculated from Eq. (14). The curve has a zero slope when $q a>1.5$, and the limit is $\omega_{d}=0.79 \omega_{a}$.

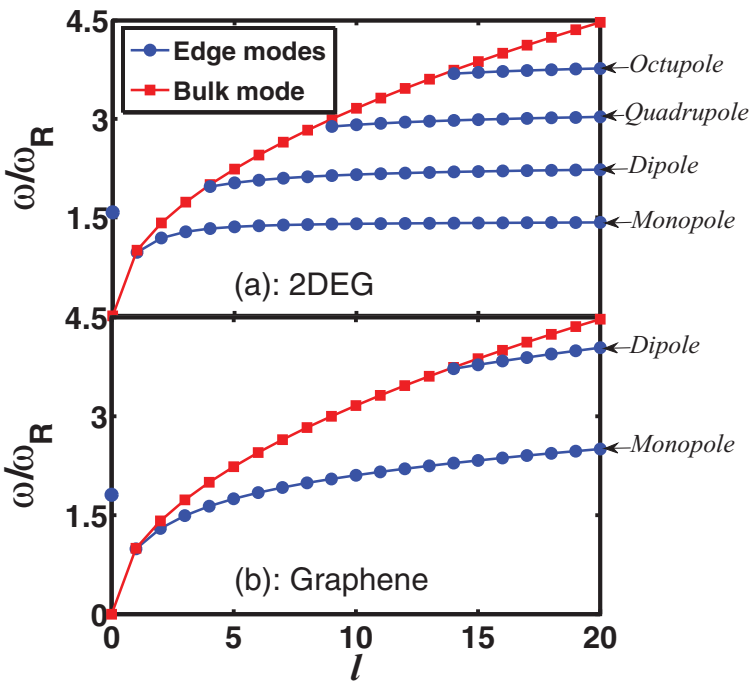

FIG. 4. (Color online) Edge plasmon dispersion in disk with edge layer width $a=0.2 R$ for 2DEG and graphene calculated from Eqs. (16) and (18). For $l=0$ only the lowest mode is shown. The dots are the computed values and the lines are to guide the eye. (a) A circular 2DEG disk showing four plasmon branches and are nearly flat for large $l$. (b) Results for a corresponding graphene disk with two plasmon branches showing plasma frequencies that increase with increasing angular momentum.

Unfortunately, this method does not work for graphene as there is no corresponding 3D material.

We solve the integral Eqs. (16) and (18) numerically and to obtain the results shown in Fig. 4. We can see that the edge plasmon behavior (except $l=0$ mode) is very similar to the semi-infinite geometry discussed in Fig. 1. In fact, with large $q a$ or $l$ the edge modes are insensitive to the geometry that leads to the same properties for both structures. The numerical results very clearly show that for large $l$ the plasmon branches are nearly flat in a 2DEG, in agreement with the semi-infinite geometry. Similarly, the edge monopole mode in graphene disk is given by $\omega \propto l^{1 / 4}$ for large $l$. Numerical results in Fig. 5 clearly shows $\omega / l^{1 / 4}$ approaches the constant $1.18 \omega_{R}$ for large $l$ in graphene disk with edge layer width $a=0.2 R$.

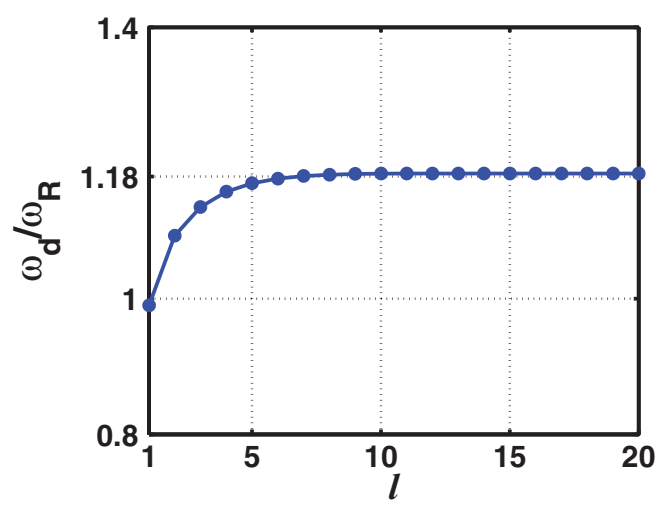

FIG. 5. (Color online) The frequencies $\omega_{d}=\omega / l^{1 / 4}$ calculated from Eq. (18) with $a=0.2 R$. The dots are numerically obtained values and the lines are to guide the eye. The curve is very nearly flat for $l>10$ and approaches to $1.18 \omega_{R}$. 

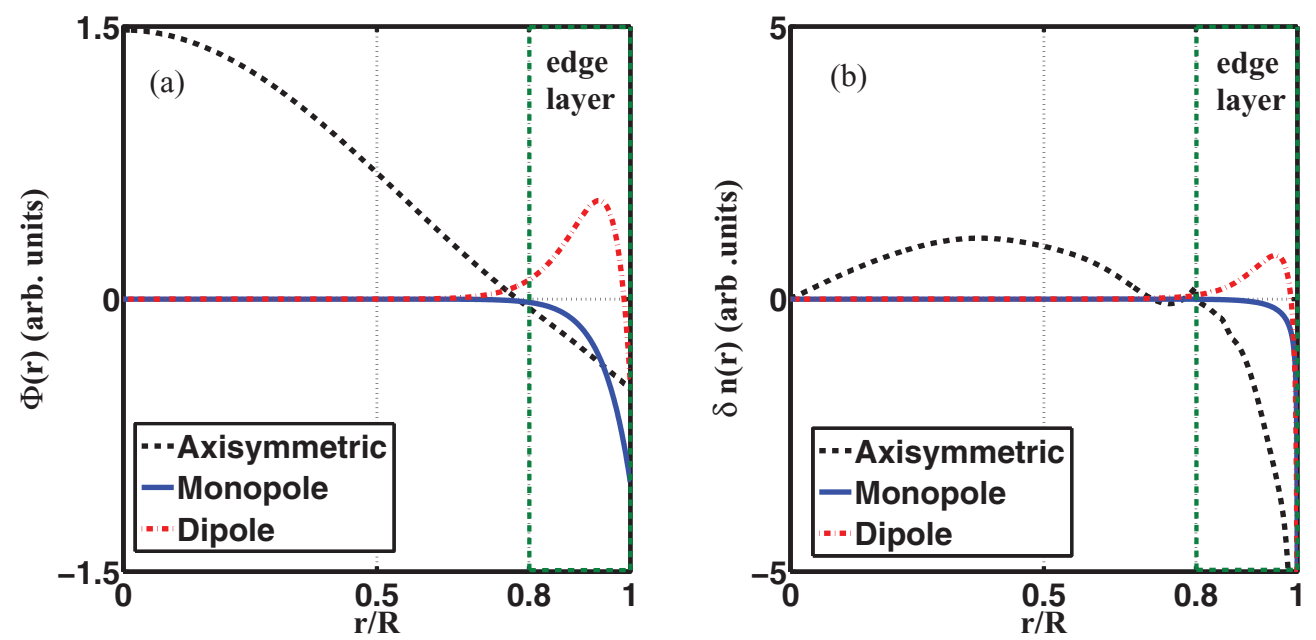

FIG. 6. (Color online) (a) Radial potential $\Phi(r)$ and (b) electron density fluctuation $\delta n(\mathbf{r})$ of three lowest plasmon modes in graphene disk at width $a=0.2 R$. Axisymmetric mode $(l=0)$ continuously extend to the center of the disk, and monopole and dipole modes at $l=18$ mostly localize in the edge layer both for potential and electron density fluctuation.

The $l=0$ axisymmetric modes must have at least one node in the radial direction due to charge conservation. They correspond to charge slushing radially in the disk and cannot be characterized as edge modes. Instead, they are analogous to the radial breathing mode (RBM) that plays on important role in carbon nanotubes. ${ }^{49-51}$ There are several axisymmetric modes characterized by the number of nodes in the radial part, and in Fig. 4 we only show the lowest mode. The $l=0$ modes extend throughout the disk as is evident from Fig. 6 where we plot the radial potential and electron density fluctuation of the lowest $l=0$ mode together with those of the two lowest modes with angular momentum $l=18$. The eigenfrequency of the lowest $l=0$ mode in a 2DEG disk with an abrupt edge was obtained by Fetter in Ref. 7 with the result $\omega_{l=0} \approx 1.86 \omega_{R}$.

Comparing the semi-infinite plane and the disk geometries, the main difference is that the plasma frequency for a disk is

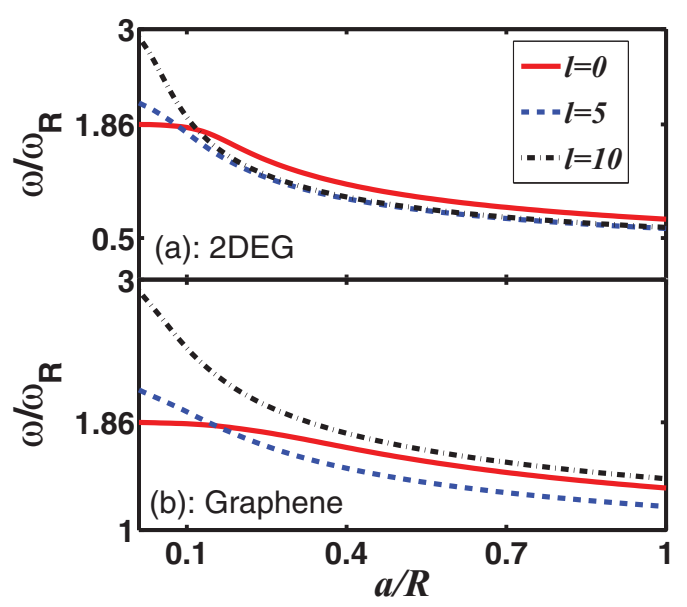

FIG. 7. (Color online) Plasma frequencies of $l=0$ mode and monopole mode for angular momenta $l=5,10$ as a function of edge layer width $a$. It is seen that a wider edge region results in lower plasma frequencies. (a) The frequencies of $l=5$ and 10 merge together for large $a$ in 2DEG, (b) in graphene, the plasmons with different angular momenta remain nondegenerate in the limit $a \rightarrow R$. quite sensitive to the edge width $a$ when $a$ becomes comparable with the disk radius $R$. Numerical results show that larger edge layer width yield lower plasma frequencies, and the lowest plasma frequencies for three values angular momenta are shown in Fig. 7 as a function of the edge width $a$. In the abrupt edge limit $a \rightarrow 0$ limit, the 2DEG and graphene disks behave similarly, and the lines with the same $l$ share the same starting points. The plasma frequencies decrease with increasing width of the edge layer, which is a consequence of a decreasing average electron density. This effect is more pronounced for the $l>0$ modes that are more tightly bound to the edge than for the $l=0$ mode that extends to the interior of the disk.

\section{CONCLUSION}

We have calculated edge plasmons in 2D semi-infinite and disk geometries both for a 2DEG and graphene, with linear edge profiles. We find that in the limit of an abrupt edge, graphene edge plasmons behave analogously with those seen in a 2DEG, but significant differences appear for structures with gradual density profiles. In particular, we find that at a given wave vector graphene supports fewer edge modes than a 2DEG. More strikingly, the edge monopole plasmon has a $q^{1 / 4}$ dispersion in both semi-infinite sheets and finite disks as opposed to the flat dispersion that is obtained for conventional 2D edge plasmons. This difference reflect the anomalous density dependence of the graphene bulk plasma frequency $\omega \propto n^{1 / 4}$ and is directly related to the unusual ac conductivity of graphene that arises from its unique band structure.

\section{ACKNOWLEDGMENTS}

We acknowledge stimulative discussions with Andreas Isacsson and Sanker Das Sarma and members of his group. This work was supported by the Swedish Research Council (VR). 
${ }^{1}$ C. C. Grimes and G. Adams, Phys. Rev. Lett. 36, 145 (1976).

${ }^{2}$ T. Ando, A. B. Fowler, and F. Stern, Rev. Mod. Phys. 54, 437 (1982).

${ }^{3}$ D. B. Mast, A. J. Dahm, and A. L. Fetter, Phys. Rev. Lett. 54, 1706 (1985).

${ }^{4}$ A. L. Fetter, Phys. Rev. B 32, 7676 (1985).

${ }^{5}$ A. L. Fetter, Phys. Rev. B 33, 3717 (1986).

${ }^{6}$ J.-W. Wu, P. Hawrylak, and J. J. Quinn, Phys. Rev. Lett. 55, 879 (1985).

${ }^{7}$ A. L. Fetter, Phys. Rev. B 33, 5221 (1986).

${ }^{8}$ J. I. Gersten, J. Chem. Phys. 77, 6285 (1982).

${ }^{9}$ R. P. Leavitt and J. W. Little, Phys. Rev. B 34, 2450 (1986).

${ }^{10}$ D. C. Glattli, E. Y. Andrei, G. Deville, J. Poitrenaud, and F. I. B. Williams, Phys. Rev. Lett. 54, 1710 (1985).

${ }^{11}$ Note that the bulk plasmons of two-dimensional systems correspond to surface plasmons of three-dimensional systems. In this article we focus on the edge plasmons which propagate along the boundaries of the systems.

${ }^{12}$ X. Xia and J. J. Quinn, Phys. Rev. B 50, 8032 (1994).

${ }^{13}$ F. C. Hoh, Phys. Rev. A 133, 1016 (1964).

${ }^{14}$ A. J. Bennett, Phys. Rev. B 1, 203 (1970).

${ }^{15}$ A. Eguiluz, S. C. Ying, and J. J. Quinn, Phys. Rev. B 11, 2118 (1975).

${ }^{16}$ N. Barberán and M. Pi, Phys. Rev. B 51, 7329 (1995).

${ }^{17}$ A. Liebsch, Phys. Rev. B 57, 3803 (1998).

${ }^{18}$ A. Liebsch, Electronic Excitations at Metal Surfaces (Plenum, New York, 1997).

${ }^{19}$ K.-D. Tsuei, E. W. Plummer, A. Liebsch, K. Kempa, and P. Bakshi, Phys. Rev. Lett. 64, 44 (1990).

${ }^{20}$ F. Moresco, M. Rocca, V. Zielasek, T. Hildebrandt, and M. Henzler, Phys. Rev. B 54, R14333 (1996).

${ }^{21}$ H. Pfnür, T. Langer, J. Baringhaus, and C. Tegenkamp (2011), e-print arXiv:1101.2768v1 [cond-mat.mes-hall].

${ }^{22}$ K. S. Novoselov, A. K. Geim, S. V. Morozov, D. Jiang, Y. Zhang, S. V. Dubonos, I. V. Grigorieva, and A. A. Firsov, Science 306, 666 (2004).

${ }^{23}$ K. S. Novoselov, A. K. Geim, S. V. Morozov, D. Jiang, M. I. Katsnelson, I. V. Grigorieva, S. V. Dubonos, and A. A. Firsov, Nature 438, 197 (2005).

${ }^{24}$ Y. Zhang, Y.-W. Tan, H. L. Stormer, and P. Kim, Nature 438, 201 (2005).

${ }^{25}$ A. H. Castro Neto, F. Guinea, N. M. R. Peres, K. S. Novoselov, and A. K. Geim, Rev. Mod. Phys. 81, 109 (2009).

${ }^{26}$ V. P. Gusynin and S. G. Sharapov, Phys. Rev. B 73, 245411 (2006).
${ }^{27}$ V. P. Gusynin, S. G. Sharapov, and J. P. Carbotte, Phys. Rev. Lett. 96, 256802 (2006).

${ }^{28}$ N. M. R. Peres, A. H. Castro Neto, and F. Guinea, Phys. Rev. B 73, 195411 (2006)

${ }^{29}$ M. Jablan, H. Buljan, and M. Soljačić, Phys. Rev. B 80, 245435 (2009).

${ }^{30}$ G. W. Hanson, J. Appl. Phys. 104, 084314 (2008).

${ }^{31}$ E. H. Hwang and S. Das Sarma, Phys. Rev. B 75, 205418 (2007).

${ }^{32}$ S. Das Sarma and E. H. Hwang, Phys. Rev. Lett. 102, 206412 (2009).

${ }^{33}$ B. Wunsch, T. Stauber, F. Sols, and F. Guinea, New J. Phys. 8, 318 (2006).

${ }^{34}$ J. Sabio, J. Nilsson, and A. H. Castro Neto, Phys. Rev. B 78, 075410 (2008).

${ }^{35}$ S. A. Mikhailov and K. Ziegler, Phys. Rev. Lett. 99, 016803 (2007).

${ }^{36}$ G. W. Hanson, J. Appl. Phys. 103, 064302 (2008).

${ }^{37}$ T. Tudorovskiy and S. A. Mikhailov, Phys. Rev. B 82, 073411 (2010).

${ }^{38}$ O. Vafek, Phys. Rev. Lett. 97, 266406 (2006).

${ }^{39}$ L. A. Falkovsky and S. S. Pershoguba, Phys. Rev. B 76, 153410 (2007).

${ }^{40}$ F. Rana, IEEE Trans. Nano Technol. 7, 91 (2008).

${ }^{41}$ V. V. Popov, T. Y. Bagaeva, T. Otsuji, and V. Ryzhii, Phys. Rev. B 81, 073404 (2010).

${ }^{42}$ F. H. L. Koppens, D. E. Chang, and F. J. García de Abajo, e-print arXiv:1104.2068v1 [cond-mat.mes-hall].

${ }^{43}$ E. G. Mishchenko, A. V. Shytov, and P. G. Silvestrov, Phys. Rev. Lett. 104, 156806 (2010)

${ }^{44}$ J. R. Williams, T. Low, M. S. Lundstrom, and C. M. Marcus, Nat. Nanotechnol. 6, 222 (2011).

${ }^{45}$ V. Shikin, S. Nazin, D. Heitmann, and T. Demel, Phys. Rev. B 43, 11903 (1991).

${ }^{46}$ N. W. Ashcroft and N. D. Mermin, Solid State Physics (Saunders, Philadelphia, PA, 1976).

${ }^{47}$ M. Abramowitz and I. A. Stegun, eds., Handbook of Mathematical Functions with Formulas, Graphs, and Mathematical Tables (US GPO, Washington, D.C., 1964).

${ }^{48}$ A. V. Klyuchnik, Y. E. Lozovik, and A. B. Operin, Phys. Lett. A 179, 372 (1993).

${ }^{49}$ A. M. Rao et al., Science 275, 187 (1997).

${ }^{50}$ A. Kasuya, Y. Sasaki, Y. Saito, K. Tohji, and Y. Nishina, Phys. Rev. Lett. 78, 4434 (1997).

${ }^{51}$ J. Kürti, G. Kresse, and H. Kuzmany, Phys. Rev. B 58, R8869 (1998). 